\title{
Carnets
}

Revue électronique d'études françaises de l'APEF

Première Série - 1 Numéro Spécial | 2009

Cultures littéraires : nouvelles performances \& développement

\section{Littérature et théâtre ou l'entre-deux. De la dramaturgie à la génétique théâtrale}

\section{Ana Clara Santos}

\section{(2) OpenEdition}

Journals

Édition électronique

URL : http://journals.openedition.org/carnets/3838

DOI : $10.4000 /$ carnets.3838

ISSN : 1646-7698

Éditeur

APEF

Édition imprimée

Date de publication : 1 juin 2009

Pagination : 221-231

\section{Référence électronique}

Ana Clara Santos, «Littérature et théâtre ou l'entre-deux. De la dramaturgie à la génétique théâtrale », Carnets [En ligne], Première Série - 1 Numéro Spécial | 2009, mis en ligne le 16 juin 2018, consulté le 19 avril 2019. URL : http://journals.openedition.org/carnets/3838 ; DOI : 10.4000/carnets.3838

\section{(c) (i) (8)}

Carnets est mis à disposition selon les termes de la licence Creative Commons - Atribution - Pas d'utilisation commerciale 4.0 International. 


\title{
LITTERATURE ET THEATRE OU L'ENTRE-DEUX \\ De la dramaturgie à la génétique théâtrale
}

Ana Clara Santos

Universidade do Algarve

avsantos@ualg.pt

\begin{abstract}
Résumé
Si on projette un regard plus attentif sur le phénomène de l'historiographie littéraire, on est vite pris par une théorisation qui privilégie une nomenclature catalographique par genre et modes littéraires. Le genre dramatique y occupe, selon les cas et les époques littéraires, une place plus ou moins anodine se profilant au sein d'une culture du canon littéraire. D'autre part, une pratique de l'enseignement littéraire et philologique tendait, dans le passé, à minimiser l'espace d'antenne accordé à la littérature dramatique. Or, paradoxalement, avec la crise récente des Langues et Littératures à l'université, on assiste de plus en plus à un renouveau envers la dramaturgie grâce auquel la littérature est relancée par le biais de l'étude de la Littérature Comparée ou de la Littérature et les Arts.
\end{abstract}

\begin{abstract}
When one takes a closer look at literary historiography, one is haunted by a set of theories which privilege a catalographic terminology based on literary modes and genres. In it, drama as a genre occupies, according to literary cases and times, a more or less unimportant place among a culture of literary canons. Furthermore, a certain practice of literary and philological teaching in the past tended to reduce the space given to drama literature. Paradoxically, the recent crisis in the academic studies of Languages and Literatures brought about a renewal of dramaturgy, thanks to which literature is once more at the center of attention, via the Comparative Literature Studies, or studies in Literature and Other Arts.
\end{abstract}

Mots-clés: Littérature, Dramaturgie, Enseignement, Recherche, Génétique théâtrale

Keywords: Literature, Drama, Teaching, Research, Theatrical genetics 
On se penchera dans cette intervention sur les rapports qui unissent la littérature et le théâtre tout en essayant de déceler, d'un côté, les liens d'ordre historiographique et esthétique et, de l'autre, les nouvelles donnes sous-jacentes à une récente pratique universitaire tant au niveau de la formation/enseignement qu'au niveau de la recherche.

\section{Le renouveau des études littéraires et théâtrales}

Longtemps enfermée dans le modèle de formation des Langues et Littératures Modernes ${ }^{1}$, l'université portugaise cherche, depuis ces dernières années, de nouvelles voies où la culture littéraire puisse trouver une place de choix. Si on fait l'exercice de comparaison entre les différentes formations offertes par les universités portugaises du nord au sud du pays, au niveau des études de premier cycle, on trouve tout d'abord la Licenciatura en Langues, Littératures et Cultures (université d'Algarve, université d'Évora, université de Lisbonne, université Nouvelle de Lisboa, université d'Aveiro, université de Porto) et celle en Langues et Littératures européennes (université du Minho). Au sein de ces formations, les étudiants peuvent choisir, comme on le sait, différents parcours :

- Études Anglaises et Françaises (Anglais-Français)

- Études Anglaises et Espagnol (Anglais-Espagnol)

- Études Anglaises et Allemand (Anglais-Allemand)

- Études Françaises (Français)

- Études Françaises et Allemandes (Français-Allemand)

- Études Françaises et Espagnoles (Français-Espagnol)

- Études Portugaises et Françaises (Portugais-Français)

- Études Portugaises et Anglaises (Portugais-Anglais)

- Études Portugaises et Espagnoles (Portugais-Espagnol)

- Études Romanes

- Langues Modernes

- Langues et Tourisme

- Littérature et Arts

Si on regarde du côté de l'enseignement de la littérature française, on constate encore un enseignement ancré sur certains genres et mouvements littéraires de l'histoire littéraire française à l'abordage chronologique par siècles. Toutefois, alors que la Littérature Francophone semble s'imposer petit à petit, en tant que discipline, à côté de la Littérature

\footnotetext{
${ }^{1}$ Rappelons, au passage, que cette formation fut associée, pendant des décennies, à la formation de professeurs, surtout des professeurs de portugais, d'anglais et de français.
} 
Française, l'introduction de disciplines comme la Littérature Générale et Comparée ainsi que la Littérature et les Arts marque, sans doute, un tournant dans l'enseignement du fait littéraire. À ce titre, voici la liste de quelques disciplines qui intègrent ces formations dans les universités en question :

- Littérature française (I-III)

- Littérature française médiévale

- Littérature française du XVIe et XVIle siècles

- Littérature française du XVIIle siècle

- Littérature française du XIXe siècle

- Littérature française contemporaine

- Littératures francophones

- Littérature française classique

- Littérature française du Romantisme et du Symbolisme

- Littérature contemporaine d'expression française

- Littérature générale et comparée

- Littérature et autres arts

- Littérature française et arts

En ce qui concerne les options, de nombreuses combinaisons sont faites entre la littérature et les sciences ou les arts tels que le cinéma et le théâtre. Dans ce domaine, la dramaturgie française apparaît comme discipline autonome ce qui constitue déjà un signe de rénovation par rapport à l'intérêt manifesté envers l'étude du phénomène théâtral :

- Littérature française et sciences

- Littérature et cinéma

- Littérature et théâtre

- Dramaturgie française

Cet intérêt se traduit effectivement par la création, ces dernières années, de Licenciaturas en Études artistiques et en Théâtre, notamment dans les universités de Coimbra, Lisbonne, Évora et Algarve. La dramaturgie, y compris la dramaturgie française, trouve alors une place qu'elle n'a jamais eu auparavant. Les auteurs du Grand Siècle, tels que Racine et Molière, ainsi que les auteurs du XXe siècle comme Artaud, Anouilh, Brecht ou Beckett sont étudiés, à juste titre, comme partie intégrante des grands classiques européens dans les disciplines suivantes: 
- Histoire du théâtre (et du spectacle)

- Théâtre et littérature

- Analyse du texte dramatique

- Dramaturgie

- Littérature étrangère (Français)

- Littérature et autres arts

- Histoire des genres dans le cinéma, la littérature et le théâtre

\section{Rapports entre le Texte et le Théâtre : un art à « deux temps »}

Si on projette un regard plus attentif sur le phénomène de l'historiographie littéraire, on est vite pris par une théorisation qui privilégie une nomenclature catalographique par genre et modes littéraires. Le genre dramatique y occupe, selon les cas et les époques littéraires, une place plus ou moins anodine se profilant au sein d'une culture du canon littéraire. D'autre part, une pratique de l'enseignement littéraire et philologique tendait, dans le passé, à minimiser l'espace d'antenne accordé à la littérature dramatique.

Or, paradoxalement, avec la crise récente des Langues et Littératures à l'université, on assiste de plus en plus, comme on vient de voir, à un renouveau envers la dramaturgie grâce auquel la littérature est relancée par le biais de l'étude de la Littérature Comparée ou de la Littérature et les Arts.

On ne peut plus continuer à nier l'évidence : les temps ont changé. L'université et l'abordage du fait littéraire et artistique aussi. II s'agit donc pour nous de présenter tout d'abord quelques études de cas concernant l'éclatement des formes et des genres au sein des dites pratiques "à deux temps" (Gouhier, 1989), de nous interroger sur le phénomène artistique de la "re-création" qui pose le problème du rapport au texte au centre des préoccupations, et finalement, d'ouvrir de nouvelles perspectives de recherche autour des "pratiques d'écriture" (Jubinville, 2007) à partir des études de génétique théâtrale menées en ce moment en France et au Canada.

À une pratique de l'enseignement littéraire et philologique centré majoritairement sur les modes poétique et romanesque, se substitue une pratique de focalisation sur la littérature dramatique. Mais les nouveautés ne s'arrêtent pas là. II faut que l'université s'adapte aux vents nouveaux et ajuste ses pratiques à un nouveau public qui, la plupart du temps, n'a pas la vocation ni les capacités de lire en langue étrangère. Je m'arrêterai, à ce propos, sur l'exemple de la Licenciatura en Études artistiques. La plupart des abordages de la dramaturgie française est faite par le biais d'une lecture sur texte traduit en langue portugaise. C'est mon expérience menée en Littérature Étrangère I (Français) autour de l'étude de la réception de certains mythes de l'Antiquité dans le théâtre français et leur 
influence sur le panorama théâtral en France et au Portugal. C'est l'expérience menée par Marta Anacleto autour de la réception de certaines pièces de Racine, Corneille et Molière sur la scène portugaise contemporaine :

La lecture de ces auteurs de théâtre, en traduction portugaise, dans le cadre de la 'Licenciatura d'Etudes Artistiques' (celle que je fais avec mes étudiants de la filière Théâtre qui ne sont pas des littéraires), ne correspond pas ainsi à un geste de soumission/dénégation linguistique mais à une attitude de 'médiation'. Le paradigme en est un autre. D'autant plus que l'on travaille, en même temps, sur le texte et sur la représentation du texte dans la scène portugaise. Ainsi, L'lllusion Comique de Corneille, mise en scène par Nuno Carinhas à partir d'une traduction de Nuno Júdice, Don Juan représenté par Ricardo Pais sous la réécriture du même traducteur, Sganarelle ou le Cocu Imaginaire, traduit par Mário Barradas et représenté à Évora (CENDREV), Bérénice mise en scène par Ricardo Pais et traduite par Vasco Graça Moura, entre autres, n'effacent point les 'classiques' français. Nuno Carinhas voit, à juste titre, ce déplacement comme une lecture possible. II s'agit, donc, de lire autrement, c'est-à-dire, de donner à lire ces textes dans le cadre d'un savoir autre qui néanmoins ne peut pas ignorer la poétique (les poétiques) dramatique du Grand Siècle et qui sert à élargir l'horizon culturel des étudiants d'Etudes Artistiques à d'autres scènes (ou à d'autres avant-scènes) culturelles (Anacleto, 2007 : 64).

Ces deux expériences, ancrées sur un abordage qui tend à privilégier une attitude de médiation entre différentes cultures et à poser les piliers d'une poétique en particulier, tendent elles aussi à exploiter ce que le philosophe Henri Gouhier a appelé un "art à deux temps".

En effet, Henri Gouhier se référant au théâtre d'avant-guerre en France et à son expérience de spectateur au cours de ces années-là, essaie de tracer, dans ses Entretiens avec Jean-Maurice de Montrémy, l'encadrement d'une philosophie de théâtre :

II y avait donc, largement répandue, une idée du théâtre considéré essentiellement comme genre littéraire, à côté du roman, du poème, etc. C'était, en gros, le point de vue de l'enseignement universitaire, ceci au profit des grands classiques, la représentation devenant une sorte de supplément plus ou moins facultatif [...]. Ce qui nous portait vers Copeau, vers Jouvet, Pitoëff, Dullin, Baty c'est qu'il y avait chez eux une protestation contre l'idée du théâtre comme genre littéraire, ceci au profit d'un théâtre plus théâtral, où la mise en scène est, elle aussi, où la représentation est inscrite dans la finalité même du théâtre, l'œuvre théâtrale étant faite pour être jouée (Gouhier, Montrémy, 2005 : 93). 
Voilà qui permet sa théorie du théâtre à deux temps. Ce qui sépare, selon lui, le théâtre d'un roman ou d'un tableau et ce qui le rapproche de la musique ou du ballet c'est que le second temps lui attribue une nouvelle existence. L'exemple de Dom Juan joué par Jouvet au Théâtre de l'Athénée suffit à illustrer l'écart existant entre le mode de la lecture dramatique et celui de la représentation théâtrale :

Pour le poème, il y a une façon d'exister qui est : il est là et puis je le lis ; le tableau est là et puis je le regarde ; mais c'est la même existence qui continue. Tandis que pour le théâtre, le second temps est un temps qui est d'exister autrement, puisqu'à ce moment-là, il faut que des acteurs viennent qui, par leur présence réelle, créent un nouveau mode d'existence. La pièce de théâtre existe, d'une part, avec son, dans son, par son texte, mais, d'autre part, elle existe aussi avec des comédiens et des comédiennes qui sont dans un certain lieu, à un certain moment, et qui par conséquent existent d'une autre façon.

Pour résumer, je dirai : je prends Dom Juan ; si je lis Dom Juan, alors vous me direz : mais vous le mettez en scène vous-même dans votre théâtre intérieur. Mais, moi, je dis : du point de vue de l'existence, à ce moment-là, ce qui existe, c'est moi en train de lire Dom Juan. Tandis que si je vais au théâtre chez Jouvet, Dom Juan c'est Jouvet présent, là, à un certain moment, tel jour à telle heure, c'est aussi dans un certain lieu, au Théâtre de l'Athénée il y avait donc là quelqu'un qui existe, une présence réelle qui était la présence réelle de Jouvet dont j'oubliais le nom qui était inscrit sur l'affiche et qui devenait Monsieur Dom Juan devant moi. (Gouhier, Montrémy, 2005 : 94-95).

À la question qu'est-ce que la représentation théâtrale, le philosophe répond par la notion de re-création qui combine, paradoxalement, les notions de fidélité et d'originalité :

C'est là une conséquence de la définition du théâtre comme art à deux temps, qui introduit dans cette définition même une contradiction entre un devoir et un droit. Le premier temps est un texte: ce texte, nous aurions dû le dire plus tôt, n'est pas seulement le texte destiné à devenir paroles, le texte desti9né à être dit ; certains auteurs décrivent la mise ne scène [...]. D'autres auteurs, au contraire, préfèrent laisser la représentation aux bons soins d'un metteur en scène expérimenté. Or dans re-création, il y a «création » et qui dit création dit une certaine originalité ; par conséquent, le metteur en scène a le droit, par là même, d'être un artiste qui invente quelque chose dans sa mise en scène. Voilà ce que j'appelle la contradiction puisque, d'un côté, il faut être fidèle et voilà que, dans un autre côté, on peut être original ; cette contradiction tient à l'essence même de l'œuvre théâtrale; alors ce qui se produit, c'est que ces deux éléments ont chacun, j'allais dire ont chacun tendance à tout envahir. Ils ont chacun tendance à réclamer une primauté ; quand j'ai débuté, c'était une protestation contre l'abus du crédit accordé au texte, et maintenant, c'est le 
contraire, c'est l'abus accordé au metteur en scène. Et alors, comment concilier tout cela ? Eh bien, je crois qu'il n'y a pas de théorie ; la critique dramatique est une casuistique ; ce sont des cas (Gouhier, Montrémy, 2005 : 96-97).

\section{De la génétique littéraire à la génétique théâtrale}

Or c'est justement sur ces notions de fidélité et d'originalité réclamées lors de la recréation de toute œuvre qu'on voudrait se fixer afin de lancer ici un nouveau défi au niveau de la recherche. Ce nouveau défi se situe au niveau des études de génétique littéraire et théâtrale, très peu développées au Portugal et qui nous permettront, non seulement de voyager du texte à la scène, mais aussi de nous interroger sur le texte pour la scène.

Mais qu'est-ce que la génétique et quels sont ses enjeux ?

On fait le plus souvent remonter à l'aventure de la génétique à l'expérience de l'équipe de Heine. Effectivement, l'héritage germanique avec cette première expérience au cours des années 60 , celle de la classification et édition des Fonds Heine transféré de Jérusalem à la BNF à Paris, ouvrira les portes à des expériences plus vastes dans le domaine de la recherche avec, notamment, l'entrée des manuscrits, brouillons, ébauches des œuvres littéraires sur la place publique, dans les Archives et les bibliothèques publiques et, surtout, avec la constitution d'équipes de recherche comme celles au CNRS et à l'ITEM.

Qu'on se souvienne, pour la mise en place de cette école, des publications parues au cours des années 70 comme Les Techniques de laboratoire dans l'étude des manuscrits (CNRS, 1974), Transcription, édition, signification (Presses de l'ENS, 1976) et les Essais de critique génétique de Louis Hay publiés aux éditions Flammarion en 1979. Qu'on se rappelle encore la création, en 1982, de l'Institution des Textes et Manuscrits Modernes (ITEM) et la Revue Genesis, dix ans plus tard. L'enjeu était de taille : connaître, analyser, interpréter et éditer les mécanismes et cheminements de "l'écriture en train de se faire"2. De ce point de vue, on peut dire que la notion de "productivité" du texte introduite par Roland Barthes ou celle de "dissémination" développée par Jacques Derrida sont incontestablement rattachées à la recherche génétique et à l'acception de la "troisième dimension de la littérature" de Louis $\mathrm{Hay}^{3}$, c'est-à-dire, celle du temps de son écriture. Visant à déceler les conditions de production d'une œuvre ou, si on veut, à étudier la "variation des états" ainsi que la "mobilité complexe et la stabilité précaire des formes", il faut, comme nous le dit Jacques Neefs, "chercher à comprendre des processus d'invention intellectuelle et esthétique qui, à travers telles activités particulières, propres à une œuvre ou à un groupe d'œuvres, peuvent caractériser un genre, un temps, une activité culturelle." (Neefs, $1988: 21$ ).

\footnotetext{
2 Tel est le titre de la revue Genesis.

${ }^{3}$ Cette notion donne le titre à un article de Louis Hay, "Die dritte Dimension der Literatur", publié dans la revue Poetica, 16, Amsterdam, Heft 3-4, 1984, pp. 307-323.
} 
Les écrivains eux-mêmes commencent à s'intéresser à ces questionnements sur leur propre activité et à léguer leurs manuscrits aux centres de recherche et aux bibliothèques. Rappelons le cas très connu d'Aragon qui, en 1976, cède ses brouillons au CNRS avec ces propos :

Ne fallait-il pas mettre à la disposition de ceux qu'on appelle les chercheurs non seulement l'écrit figé par la publication, mais le texte en devenir, saisi pendant le temps de l'écriture, avec ses ratures comme ses repentirs, miroirs des hésitations de l'écrivain comme des manières de rêverie que révèlent les achoppements du texte. Le champ de nos rapports, j'entends de l'écrivain et du chercheur, est celui de l'écrit (...) dans ce champ-là nous sommes, les uns et les autres, curieux, d'une curiosité naturelle, à la fois de connaître et comprendre la recherche aussi bien de la chose à écrire que la recherche qui s'en fait (Aragon, $1979: 8-9)$.

Ces propos d'Aragon renvoient, bien sûr, à la révolution poétique accomplie par les écrivains du tournant du XIXe siècle et du XXe siècle, à savoir, Mallarmé, Valéry, Proust ou Gide car l'enjeu était devenu autre depuis les origines de la poétique mallarméenne, énoncée en 1864 comme "peindre non la chose, mais l'effet qu'elle produit" (Mallarmé, 1995 : 206). Définie selon la formule de Valéry "le faire comme principal et la chose faite comme accessoire", on approche une nouvelle esthétique, celle de l'œuvre en acte. Mais cette nouvelle conception de l'écriture, souscrite par les écrivains eux-mêmes, ne facilite qu'en apparence la tâche du critique littéraire. Écoutons les mots savants d'Henri Mitterand :

L'essentiel est de ne se payer ni de mots, ni de modes, ni de théories, ni d'idées toutes faites, mais d'avoir pour le texte un respect de philologue - et ce n'est pas peu dire -, et de se rappeler que la critique génétique est un exercice asymptotique : on a beau se rapprocher toujours davantage de la ligne où l'on croit voir surgir la vérité de l'œuvre, toujours celle-ci reste hors de saisie, préservant la part du génie et de l'art. Mais il y a quelque mérite à tenter d'aller aussi loin que possible en direction de ce cœur inaccessible. On peut y prendre aussi beaucoup de plaisir (Mitterand : 1993).

Or, là-dessus, lorsqu'on passe de l'art littéraire à l'art théâtral, le processus devient encore plus complexe car si l'enjeu était de taille pour la génétique littéraire il l'est encore davantage pour la génétique théâtrale qui a l'ambition de cerner cet "art à deux temps". Comment analyser la genèse d'une pièce, acte éphémère, caractérisé par une mobilité inhérente, qui n'a de réalisation que le moment de sa représentation ?

Malgré les écueils d'une telle approche, une chose est sûre. À l'image de ce qui s'est vérifié pour la génétique littéraire, seul le développement des recherches de génétique 
théâtrale permettront une prise de conscience du phénomène de façon à contribuer à la conservation d'archives théâtrales. Les uns et les autres seront, sûrement, plus conscients de la nécessité de conserver les traces fragiles de la naissance d'un spectacle, par le biais, notamment, de journaux de bord, de livres de régie, de captations de répétitions, de croquis pour le décor, etc. On le sait, dans ce domaine, l'analyse génétique prend axe sur la reconstruction des étapes successives de l'élaboration du spectacle, du texte écrit au texte scénique, avec le passage obligé par le cabinet d'écriture privé du dramaturge et par la sphère publique de la circulation des biens culturels du monde de l'édition, celui de l'appropriation du texte par les acteurs, ou encore, celui de la relecture opérée par le metteur en scène qui le met sous les yeux du public. Bien que l'existence du texte écrit en vue de la scène parcourt, à peu près, les mêmes phases génétiques qu'un texte en prose ou en poésie, la composante scénique, partie intégrante du processus, lui confère une spécificité particulière qui, au départ, le projette vers de nouveaux dédoublements scripturaux et de nouvelles réécritures constituant, ainsi, ce que Anne Ubersfeld appelle "une matrice de la représentativité".

En effet, l'impact de la première et la réception du texte/spectacle peuvent produire de nouveaux réajustements et un nouveau travail de remaniement. Là aussi, les exemples abondent. C'est Genet qui écrit à son éditeur Marc Barbezat dans une lettre datée du 26 octobre 1959 au sujet du Balcon en lui disant de ne pas inclure, dans l'édition, la mention "édition définitive" car il a l'intention de retravailler la pièce jusqu'à sa mort. C'est la négociation établie entre les dramaturges et certains médiateurs, passeurs entre le monde du texte littéraire ou dramatique et le monde de la scène théâtrale, notamment les metteurs en scène, dans un processus qu'on pourrait appeler d' "écriture à deux mains". On se souvient des couples parfaits de Jean Giraudoux - Louis Jouvet pour la mise en scène et la réécriture des pièces Ondine (1939), Amphitryon 38 (1929), La Folle de Chaillot (1945) ; de Jean Genet - Roger Blin pour Paravents (1966) ; de Paul Claudel - Jean-Louis Barrrault pour Le soulier de satin (1943) et le Partage de midi (1948).

L'effervescence des études menées à l'étranger, notamment en France et au Canada, dans le domaine des études de génétique théâtrale tendent bien à montrer, d'un côté, l'importance de l'abolition des tensions entre la littérature et le théâtre, entre le texte et le spectacle et, de l'autre, la nécessité de raviver l'approche de ces phénomènes littéraires et artistiques en tant que phénomènes à trois temps entre ce qui est écrit, ce qui est dit/joué et ce qui est vu. Cette complexité est bien ressentie comme un défi pour les chercheurs qui courent après ces œuvres en mouvement toujours indissociables de «ce jeu énergétique des écritures » et de ces re-créations : 
Alors que les manuscrits de poèmes et de romans, sous l'effet d'une force centripète, semblent continuellement ramener l'intérêt de l'analyse vers un noyau central composé du couple indissociable auteur/œuvre, les manuscrits de théâtre se trouvent comme emportés par une force centrifuge qui les pousse en permanence vers l'extérieur dans une expansion qui fragmente les textes et diversifie les scripteurs. Ces derniers participent soit du pôle de l'écrit des dialogues et de ses discours d'accompagnement, soit de celui de la création scénique sans toutefois qu'entre ces deux extrêmes ne cesse une interaction toujours décisive.

Ces conditions particulières poussent la critique génétique à élargir ses frontières et à réajuster ses moyens d'investigation en tenant compte de chacun des éléments issu des traces manuscrites, fût-il ténu et fugace. Elles l'obligent aussi à rechercher de nouveaux modus operandi pour recomposer l'unité fragmentée de l'œuvre théâtrale, à poser autrement de nouvelles questions quitte à multiplier les clés des codes herméneutiques et à en éprouver les incertitudes, la complexité et e péril. (Thomasseau, $2005:$ 118).

\section{En guise de conclusion}

Par tout ce qui précède, la convocation et l'actualisation de certaines pratiques d'écriture théâtrales qui tiennent en compte les pratiques à "deux temps" constituent la voie trouvée pour la survie de la culture dramaturgique française dans certaines formations universitaires au Portugal.

Ce renouveau des études littéraires et théâtrales françaises à l'université au Portugal doit favoriser la recherche de nouveaux terrains d'entente et d'investigation susceptibles de développer ce champ d'études dans les prochains temps. Ce Forum est, à ce titre, depuis hier, un lieu de confrontation de nouvelles perspectives et de nouveaux défis qui permettront d'atteindre nos objectifs afin de maintenir la survie et l'excellence des études littéraires françaises dans l'enseignement supérieur portugais.

L'incitation à la recherche dans de nouveaux domaines, notamment dans celui que j'ai présenté ici, c'est-à-dire, celui de la génétique théâtrale, qui fait l'objet d'un nouveau projet de colloque à réaliser au mois de décembre 2009, au sein du Centre d'Études de Théâtre de l'université de Lisbonne, peut contribuer aussi, comme on l'espère, à relancer la recherche dans le domaine des études littéraires et artistiques au Portugal. 


\section{Bibliographie}

Anacleto, Marta, Santos, Ana Clara, Marinho, Cristina (2007). "La dramaturgie française et les études théâtrales au Portugal". In: Espaces de la Francophonie en débat,Forum Apef 2006 [consulté le 15 septembre 2008 ] <URL: http://www.apef.org.pt/actas2006/MAC122006.pdf>, pp.61-71.

Aragon, Louis (1979). "D’un grand art nouveau, la recherche". In Hay, Louis (éd.), Essais de critique génétique. Paris : Flammarion.

Glénisson, J., Hay, L. (1974). Les Techniques de laboratoire dans l'étude des manuscrits. Paris: CNRS

Gouhier, Henri (1989). Le théâtre et les arts à deux temps. Paris : Flammarion.

Gouhier, Henri, Montrémy, Jean-Maurice de (2005). Cinq entretiens avec Jean-Maurice de Montrémy. Paris : Vrin.

Grésillon, Almuth, Hechenblaickner, Angelika et al. (éd.). De la genèse du texte littéraire. Manuscrit, auteur, texte, critique, Actes du colloque franco-soviétique ITEM /CNRS, Paris : Institut Gorki de Littérature Mondiale. Académie des Sciences, Moscou- Paris, INALCO, 8-9 octobre 1987.

Hay, Louis (éd.) (1979). Essais de critique génétique. Paris : Flammarion.

Hay, Louis (1984). "Die dritte Dimension der Literatur". In Poetica, 16. Amsterdam, Heft 3-4, pp. 307323.

Hay, Louis (éd.) (1993). Les Manuscrits des écrivains. Paris : CNRS/Hachette.

Jubinville, Yves (2007). 'Le partage des voix : approche génétique de la langue dans les dramaturgies québécoises contemporaines". In Études Françaises, vol. 43, oํ 1, pp. 101-119.

Mitterand, Henri. (1993). 'Archives zoliennes: la genèse de La Faute de l'Abbé Mouret. Étude d'un dossier manuscrit". In Hay, Louis (éd.). Les Manuscrits des écrivains. Paris : CNRS/Hachette.

Neefs, Jacques (1988). "La critique génétique: I'histoire d'une théorie". In Grésillon, Almuth, Hechenblaickner, Angelika et al. (éd.). De la genèse du texte littéraire. Manuscrit, auteur, texte, critique, Actes du colloque franco-soviétique ITEM /CNRS, Paris: Institut Gorki de Littérature Mondiale. Académie des Sciences, Moscou- Paris, INALCO, 8-9 octobre 1987. Tusson : Du Lérot, pp. 11-22.

Thomasseau, Jean-Marie (2005). "Les manuscrits de théâtre. Essai de typologie". In Littérature, no 138, Théâtre : le retour du texte?, pp. 97-118.

Ubersfeld, Anne (1982). Lire le théâtre. Paris : Messidor/Éditions Sociales.

(1976). Transcription, édition, signification. Paris : Presses de l'ENS.

(1992). Genesis. Revue Internationale de Critique génétique. L'écriture en train de se faire.

(2006). Genesis. Revue Internationale de Critique génétique. №. 26, Théâtre. 